\title{
A case of hematuria and vomiting in the emergency room: Never forget the emphysematous pyelonephritis
}

\author{
Erika Poggiali, ${ }^{1}$ Fabio Tansini, ${ }^{1}$ Konstantinos Christodoulakis, ${ }^{2}$ Manuela Giovini, ${ }^{3}$ \\ Andrea Magnacavallo, ${ }^{1}$ Andrea Vercelli ${ }^{1}$
}

\author{
${ }^{1}$ Emergency Department; ${ }^{2}$ Urology Unit; ${ }^{3}$ Intermediate Care Unit, Emergency Department, “Guglielmo da \\ Saliceto" Hospital, Piacenza, Italy
}

\begin{abstract}
We describe the case of a 68 -year-old man with a known history of hypertension and diabetes mellitus presented to our Emergency Department, complaining of hematuria and vomiting in the last 12 hours, stypsis and urinary incontinence in the last week, and worsening hyporexia in the last 6 months. Bedside ultrasound documented a slight right pleural effusion with B lines in the middle and basal right field, gastrectasis, dilated fluid-filled bowel loops, potential signs of gas in the upper right quadrant, grade 3 bilateral hydronephrosis, and bladder globe. Abdominal CT scan confirmed the bilateral hydroureteronephrosis and showed the right kidney with Emphysematous Pyelonephritis (EPN) with extension into the perinephric and muscular planes for $24 \mathrm{~cm}$, and
\end{abstract}

Correspondence: Erika Poggiali, Emergency Department, "Guglielmo da Saliceto" Hospital, Via Giuseppe Taverna 49, Piacenza, Italy.

Tel.: +39.0523.303044

E-mail: poggiali.erika@gmail.com

Key words: Emphysematous pyelonephritis; hematuria; acute renal failure; urosepsis; hydroureteronephrosis.

Contributions: EP and FT collected details of the case and drafted the manuscript. EP, KC, and MG cared for the patient. AM e AV critically revised the manuscript. All authors approved the final version and stated the integrity of the whole work.

Conflicts of interest: The authors declare no conflict of interest. EP is a member of the editorial board of Emergency Care Journal.

Availability of data and materials: All data underlying the findings are fully available.

Ethics approval and consent to participate: As this was a descriptive case report and data was collected without patient identifiers, ethics approval was not required under our hospital's Institutional Review Board guidelines.

Informed consent: The patient provided consent for the access to medical records at the time of admission.

Received for publication: 11 May 2021.

Revision received: 16 August 2021.

Accepted for publication: 16 August 2021.

This work is licensed under a Creative Commons Attribution 4.0 License (by-nc 4.0).

(C) Copyright: the Author(s), 2021

Licensee PAGEPress, Italy

Emergency Care Journal 2021; 17:9847

doi:10.4081/ecj.2021.9847 initial EPN in the left kidney. A RT-PCR nasopharyngeal swab for SARS CoV-2 was negative. A diagnosis of ileum paretic, acute renal failure and urosepsis due to EPN was made.

\section{Case Report}

A 68-year-old man affected by hypertension and well controlled diabetes mellitus presented to our Emergency Department with complaints of painless gross hematuria and vomiting in the last 12 hours, progressive and worsening hyporexia, dehydration, asthenia, weight loss in the last 6 months, and a new onset of stypsis and urinary incontinence in the last week. His medications included salic acid, bisoprolol, Olmesartan and repaglinide. He denied dysuria, cough, fever, or chills. His blood pressure was $120 / 60 \mathrm{mmHg}$, heart rate 75 beats $/ \mathrm{min}$, temperature $36^{\circ} \mathrm{C}$, respiratory rate 22 breaths $/ \mathrm{min}$ and he had an oxygen saturation of $95 \%$ at room ambient and $98 \%$ on $2 \mathrm{~L} \mathrm{O}_{2}$ delivered via nasal prongs. On examination he had severe signs of dehydration, including dry skin and oral mucous membranes and flat neck veins, and cachexia with muscle hypotrophy and weakness involving both the legs and the arms. Abdomen was distended with mild tenderness to palpation of his right upper quadrant with no Blumberg's sign. The pulmonary flow murmur was absent in the right lower lobe.

Sinus rhythm and Right Bundle Branch block (RBB) was detected on ECG. Point-of-care ultrasound (PoCUS) documented a slight right pleural effusion with $\mathrm{B}$ lines in the middle and basal right field, gastrectasis, dilated fluid-filled bowel loops with hyperechoic spots of gas moving within the fluid and absent peristalsis, potential signs of gas in the upper right quadrant and grade 3 bilateral hydronephrosis with bladder globe (Figure 1); inferior vena cava was completely collapsed, as sign of volume depletion.

Venous blood gas analysis showed a metabolic acidosis $(\mathrm{pH}$ $7.29, \mathrm{HCO}_{3} 16.2 \mathrm{mmol} / \mathrm{L}$ ) with acute renal failure (creatinine 5 $\mathrm{mg} / \mathrm{L})$, hyperkaliemia $(5.6 \mathrm{mEq} / \mathrm{L})$ and an increased anion gap (21.4 mmol/L), moderate anemia (Hb $9.1 \mathrm{~g} / \mathrm{dL}$, Hct 27.8\%), and elevated lactate (38 mg/dL, normal value 5-15). Glucose and bilirubin were normal.

Firstly, we inserted a nasogatric tube and we aspirated $600 \mathrm{~mL}$ of fecaloid material, then we placed a foley catheter that drained $500 \mathrm{~mL}$ of dark urine without clots. Urine dipstick showed turbid appearance with hematuria and proteinuria. We removed the foley catheter and placed a three-way urinary catheter. Manual bladder irrigation and empiric broad-spectrum intravenous antibiotic therapy with Piperacillin/Tazobactam at dose of 4.5 gr were immediately started, and then adjusted to the degree of acute renal impairment. The patient was hydrated with intravenous glucose $5 \%$ solution $500 \mathrm{~mL}$, sodium chloride $0.9 \% 1000 \mathrm{~mL}$ and sodium bicarbonate $8.4 \% 500 \mathrm{~mL}$, and he was transfused with one unit of blood without side effects. PoCUS documented a persistent bilateral grade 2 hydronephrosis even with empty bladder. Laboratory investigations are reported in Table 1, and confirmed acute renal 
failure with hyperkalemia, significantly increased white blood cell count with neutrophilia and elevated C-reactive protein, and moderate microcytic anemia. Liver function and coagulation times were within normal limits. In addition, urine analysis showed turbid appearance with obvious hematuria, glycosuria $(50 \mathrm{mg} / \mathrm{dL}$, normal value $0-15)$ and proteinuria $(100 \mathrm{mg} / \mathrm{dL}$, normal value 0 $30)$ with an increased urine albumin-to-creatinine ratio $(>300$ $\mathrm{mg} / \mathrm{g}$, normal value $<30$ ). A RT-PCR nasopharyngeal swab for SARS-CoV-2 was negative. Serology test revealed a previous SARS-CoV-2 infection (IgG positive, IgM negative). Legionella and Streptococcus pneumoniae urine antigens resulted absent.

High resolution chest CT scan confirmed the slight right pleural effusion, showed bilateral bronchial secretions with the com-
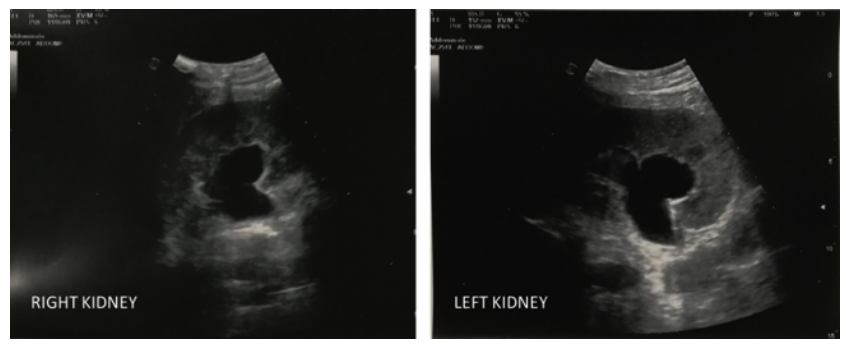

Figure 1. Bilateral grade 3 hydronephrosis. plete segmental bronchial obstruction of the right lower lobe, and excluded interstitial pneumoniae. Urgent abdomen CT scan without contrast documented a bilateral hydroureteronephrosis and showed the right kidney with Emphysematous Pyelonephritis $(\mathrm{EPN})$ with extension into the perinephric and muscular planes for $24 \mathrm{~cm}$, and initial EPN in the left kidney (Figure 2). In addition, CT scan revealed distended small bowel loops in the hypogastric

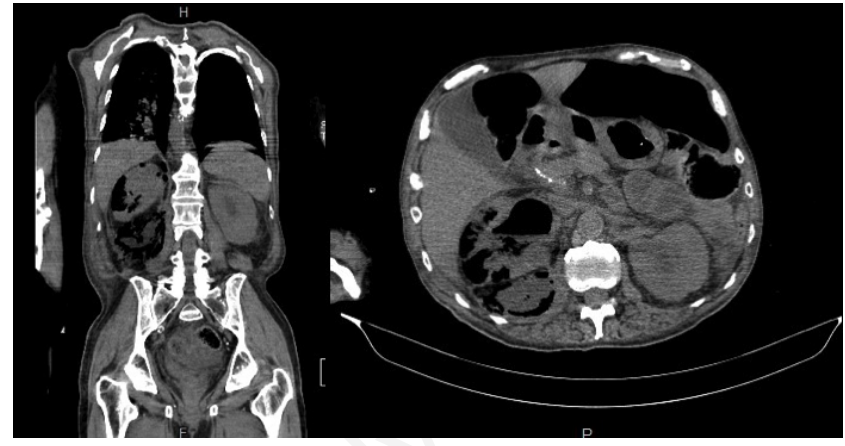

Figure 2. Abdomen CT scan without contrast showing enlarged right kidney with a gas collection within the renal parenchyma with extension into the perinephric and muscular planes for 24 $\mathrm{cm}$, and distended small bowel loops (coronal and transverse section).

Table 1. Patient's laboratory findings at admission and during recovery. WBC, white blood cells; RBC, red blood cells; Hb, hemoglobin; MCV, mean cell volume; MCH, mean cell hemoglobin; PLT, platelet; PT, prothrombin time; PTT, partial thromboplastin time; CRP, C-reactive protein; PCT, procalcitonin; LDH, lactate dehydrogenase; ALT, alanine transaminase; AST, aspartate transaminase; GGT, gamma glutaryltransferase. ND, not done. Normal range values are in brackets, altered values in bold.

\begin{tabular}{|c|c|c|}
\hline & At admission & Recovery day 3 \\
\hline WBC $\left(4-10 \times 10^{9} / \mathrm{L}\right)$ & 11.27 & 13.95 \\
\hline Neutrophil Count $\left(2-8 \times 10^{9} / \mathrm{L}\right)$ & 10.65 & 12.57 \\
\hline Lymphocyte Count (1.5-4 x 109/L) & 0.35 & 0.97 \\
\hline $\mathrm{RBC}(4.3-5.7 \times 106 / \mathrm{L})$ & 2.94 & 3.66 \\
\hline $\mathrm{Hb}(13.2-17.3 \mathrm{~g} / \mathrm{dL})$ & 8.5 & 10.6 \\
\hline MCV (82-98 fL) & 87.1 & 86.6 \\
\hline MCH (27-32 pg) & 25.6 & 29 \\
\hline PLT Count $\left(150-450 \times 10^{9} / \mathrm{L}\right)$ & 156 & 37 \\
\hline PT-INR & 1.57 & 1.53 \\
\hline PTT sec & 30.6 & 33 \\
\hline Glucose (74-100 mg/dL) & 112 & 189 \\
\hline $\mathrm{CRP}(<0.5 \mathrm{mg} / \mathrm{dL})$ & 25.97 & 28.85 \\
\hline PCT $(<0.5 \mathrm{ng} / \mathrm{mL})$ & ND & 92 \\
\hline Fibrinogen $(150-400 \mathrm{mg} / \mathrm{dL})$ & ND & 656 \\
\hline LDH (0-248 U/L) & 666 & ND \\
\hline Creatinine (0.6-1.2 mg/dL) & 5.11 & 4.27 \\
\hline Blood urea nitrogen (10-50 mg/dL) & 276 & 241 \\
\hline Potassium (3.5-5.0 mEq/L) & 6.2 & 6.6 \\
\hline Sodium (135-146 mEq/L) & 136 & 138 \\
\hline Calcium (8.1-10.4 mg/dL) & ND & 7.16 \\
\hline Fosfate $(2.6-4.5 \mathrm{mg} / \mathrm{dL})$ & ND & 5.8 \\
\hline AST (10-37 U/L) & 48 & 41 \\
\hline ALT (10-37 U/L) & 27 & 25 \\
\hline GGT (7-40 U/L) & ND & 40 \\
\hline Cholinesterase (4620-11500 U/L) & ND & 2695 \\
\hline Total bilirubin (0.1-1.1 mg/dL) & 0.17 & 1.2 \\
\hline
\end{tabular}


region, with diffuse coprostasis, in absence of air-fluid levels.

Urology consultation was obtained in the emergency room. Given the extensive involvement of the renal parenchyma and bilateral collecting systems, the urologist decided to do an immediate right percutaneous nephrostomy using ultrasound guidance, along with supportive care. After the procedure, the patient was admitted to the subintensive care unit with a diagnosis of acute renal failure and ileum paretic owing to bilateral EPN. Urine and blood cultures obtained on presentation to the Emergency Department quickly grew out multi-resistant Escherichia Coli. Unfortunately, the patient died from septic shock after 3 days of hospitalization. Laboratory data during the recovery are reported in Table 1.

\section{Discussion}

EPN is a rare but severe life-threatening acute necrotizing infection of the kidney, predominantly in patients with diabetes $(70-90 \%)^{1}$ and/or with urinary tract obstruction. ${ }^{2}$ It is more common in females than males, particularly in female patients with poorly controlled diabetes, ${ }^{3}$ and only in $10 \%$ of cases involves both the kidneys. ${ }^{4,5}$ EPN is characterized by gas production and accumulation in the renal parenchyma, collecting system, or perinephric tissues. ${ }^{6,7}$ Over $90 \%$ of the reported cases are due to Escherichia Coli, but other microbes can be Proteus mirabilis, Klebsiella pneumonia, Pseudomonas aeruginosa, Bacteroides fragilis, Aerobacter aerogenes, Candida albicans, and Cryptococcus neoformans. ${ }^{6,8-10}$ The first case of EPN was described in 1898 by Kelly and MacCallum as "pneumaturia" for the detection of gas in the urinary tract during cystoscopy. ${ }^{11}$ The pathogenesis of EPN is still unclear. Several authors reported hyperglycemia, altered tissue perfusion, and impaired immune response as contributors to the development of EPN. .,12,13 Gas-production infections of the kidney and urinary tract can be due to EPN, emphysematous pyelitis, and emphysematous cystitis. ${ }^{14}$ It is extremely important to early recognize and diagnose these conditions because of their life-threatening potential. Patients with EPN can rapidly develop septic shock and multiple organ failure, with a mortality rate of up to $30 \%$. Unfortunately, signs and symptoms of EPN are unspecific and can include fever, nausea, pain in the abdomen, in the flanks or back. ${ }^{15-17}$ Rare cases of pneumomediastinum and subcutaneous emphysema, or multiple septic emboli to the brain, lungs, and liver have been reported as unusual presentation of EPN. ${ }^{18,19}$ Acute renal failure, thrombocytopenia, sepsis, altered mental status, including delirium, and need for hemodialysis are considered as poor prognostic risk factors. ${ }^{6,20} \mathrm{EPN}$ is a radiological diagnosis. Abdominal CT scan is the gold standard technique for early diagnosis and staging. ${ }^{21-22}$ It can define the extent and quantification of the gas, and any destruction of the renal parenchyma. Huang-Tseng classified EPN into four stages based on CT findings: stage 1 is classified as gas in collecting system only, stage 2 as gas in renal parenchyma only, stage 3 as gas in renal parenchyma with extrarenal extension - 3 a extension of gas or abscess to perinephric space, and $3 \mathrm{~b}$ extension of gas or abscess to pararenal space, and stage 4 as bilateral emphysematous pyelonephritis or solitary kidney with emphysematous pyelonephritis, as in our patient. ${ }^{6}$ The key is to identify EPN as early as possible so that less invasive interventions can be successfully used. Control of diabetes, correction of diabetic ketoacidosis, systemic antibiotics and hydration are mandatory in all the cases. Patients in classes 1 and 2 can be treated conservatively with prompt hydration, antibiotics, and Percutaneous Catheter Drainage (PCD) or stent. Bilateral PCD should be performed in patients in class 4 ; immediate nephrectomy for class 3 patients with more than two risk factors; and nephrectomy for all cases where PCD fails. ${ }^{23,24}$ As reported in literature, PCD is now the most appropriate strategy and the gold standard in management of EPN. ${ }^{25-28}$ Our patient has a 4 class EPN with acute renal failure and sepsis, which are risk factors associated with poor prognosis. He presented also an adynamic ileus, which can be caused by several conditions, including sepsis, especially for Gram-negative bacteria, as in our case, and he was in extremely poor general condition, making the patient an unlikely surgical candidate. An attempt at PCD has been proposed by the urologist even if the risks associated with the procedure were substantial.

\section{Conclusions}

The diagnosis of EPN is a challenge for the emergency clinicians by virtue of its rarity and unspecific clinical signs and symptoms, with a higher mortality than conventional cases of pyelonephritis. EPN should be suspected in multimorbid patients with a poor controlled diabetes mellitus, who present with recurrent pyelonephritis or relapsing urosepsis, generally caused by $E$. Coli species not responding to antibiotics. Non-contrast CT abdominal imaging is mandatory for the diagnosis and the classification of EPN. Severe sepsis, delirium, thrombocytopenia, or need for hemodialysis are negative prognostic factors. Given the multiple comorbid nature of patients who present with EPN, the management of EPN should be based on a multidisciplinary team decision-making, inclusive of urologists, nephrologists, radiologists, and microbiologists. A conservative approach using percutaneous drainage and microbiology-guided intravenous antibiotics is currently considered as preferable to bilateral nephrectomy.

\section{References}

1. Pontin AR, Barnes RD, Joffe J, Kahn D. Emphysematous pyelonephritis in diabetic patients. Br J Urol 1995;75:71-4.

2. Tang HJ, Li CM, Yen MY et al. Clinical characteristics of emphysematous pyelonephritis. J Microbiol Immunol Infect 2001;34:125-30.

3. Song Y, Shen X. Diabetic ketoacidosis complicated by emphysematous pyelonephritis: a case report and literature review. BMC Urol 2020;20:6.

4. Shokeir AA, El-Azab M, Mohsen T, El-Diasty T. Emphysematous pyelonephritis: a 15-year experience with 20 cases. Urology 1997;49:343-6.

5. Zhang Y, Zang GQ, Tang ZH, Yu YS. Emphysematous pyelonephritis. Rev Inst Med Trop Sao Paulo 2015;57:368.

6. Huang JJ, Tseng CC. Emphysematous pyelonephritis: clinicoradiological classification, management, prognosis, and pathogenesis. Arch Intern Med 2000;160:797-805.

7. Ubee SS, McGlynn L, Fordham M. Emphysematous pyelonephritis. BJU Int 2011;107:1474-8.

8. Liao HW, Chen TH, Lin KH et al. Emphysematous pyelonephritis caused by Bacteroides fragilis. Nephrol Dial Transplant 2005;20:2575-7.

9. Christensen J, Bistrup C. Case report: emphysematous pyelonephritis caused by clostridium septicum and complicated by a mycotic aneurysm. Br J Radiol 1993;66:842-3. 
10. Hildebrand TS, Nibbe L, Frei U, Schindler R. Bilateral emphysematous pyelonephritis caused by Candida infection. Am J Kidney Dis 1999;33:E10.

11. Kelly HA, MacCallum WG. Pneumaturia. J Am Med Assoc 1898;31:375-81.

12. Mallet M, Knockaert DC, Oyen RH, Van Poppel HP. Emphysematous pyelonephritis: no longer a surgical disease? Eur J Emerg Med 2002;9:266-9.

13. Tseng CC, Wu JJ, Wang MC, et al. Host and bacterial virulence factors predisposing to emphysematous pyelonephritis. Am J Kidney Dis 2005;46:432-9.

14. Evanoff GV, Thompson CS, Foley R, Weinman EJ. Spectrum of gas within the kidney. emphysematous pyelonephritis and emphysematous pyelitis. Am J Med 1987;83:149-54.

15. Yao J, Gutierrez OM, Reiser J. Emphysematous pyelonephritis. Kidney Int 2007;71:462-5.

16. Sama S, Chandra N. Unusual presentation of emphysematous pyelonephritis. Intensive Care Med 2019;45:525.

17. Hirose Y, Kaida H. Emphysematous Pyelonephritis. N Engl J Med 2016;375:1671.

18. Wang YC, Wang JM, Chow YC, et al. Pneumomediastinum and subcutaneous emphysema as the manifestation of emphysematous pyelonephritis. Int J Urol 2004;11:909-11.

19. Bhatt B, Bhavsar B, Senanayke S, et al. Bilateral emphysematous pyelonephritis with multiple septic emboli to brain, lungs, and liver as an initial presentation of new-onset diabetes mellitus. NY Med J 2006

20. Kangjam SM, Irom KS, Khumallambam IS, Sinam RS. Role of conservative management in emphysematous pyelonephritis
- A retrospective study. J Clin Diagn Res 2015;9:PC09-11.

21. Narlawar RS, Raut AA, Nagar A, et al. Imaging features and guided drainage in emphysematous pyelonephritis: A study of 11 cases. Clin Radiol 2004;59:192-7.

22. Chen CY, Chen CJ. Images in clinical medicine. Emphysematous pyelonephritis. N Engl J Med 2014;371:e34.

23. Chan PH, Kho VK, Lai SK, et al. Treatment of emphysematous pyelonephritis with broad-spectrum antibacterials and percutaneous renal drainage: an analysis of 10 patients. J Chin Med Assoc 2005;68:29-32.

24. Mydlo JH, Maybee GJ, Ali-Khan MM. Percutaneous drainage and/or nephrectomy in the treatment of emphysematous pyelonephritis. Urol Int 2003;70:147-50.

25. Somani BK, Nabi G, Thorpe $\mathrm{P}$, et al. Is percutaneous drainage the new gold standard in the management of emphysematous pyelonephritis? Evidence from a systematic review. J Urol 2008;179:1844-9.

26. Deoraj S, Zakharious F, Nasim A, Missouris C. Emphysematous pyelonephritis: outcomes of conservative management and literature review. BMJ Case Rep. 2018;2018:bcr2018225931.

27. Karthikeyan VS, Manohar CMS, Mallya A, et al. Clinical profile and successful outcomes of conservative and minimally invasive treatment of emphysematous pyelonephritis. Cent European J Urol 2018;71:228-33.

28. Boakes E, Batura D. Deriving a management algorithm for emphysematous pyelonephritis: can we rely on minimally invasive strategies or should we be opting for earlier nephrectomy? Int Urol Nephrol 2017;49:2127-36. 